K. NIINO

KODAI MATH. J.

16 (1993), $455-468$

\title{
ON AN ULTRAHYPERELLIPTIC SURFACE WITH PICARD CONSTANT THREE
}

Dedicated to Professor Nobuyuki Suita on his 60th birthday

BY KIYOSHI NiINO

\section{$\S 1$. Introduction.}

Let $R$ be an open Riemann surface. Let $M(R)$ be the family of non-constant meromorphic functions on $R$. Let $P(f)$ be the number of values which are not taken by $f=M(R)$. The Picard constant $P(R)$ of $R$ is defined by

$$
P(R)=\sup \{P(f) ; f \in M(R)\}
$$

Then we have $P(R) \geqq 2$. The significance of the Picard constant is in the following fact: If $P(R)<P(S)$, then there is no non-constant analytic mapping of $R$ into $S$ (Ozawa [5]).

Let $R$ be the ultrahyperelliptic surface defined by

$$
y^{2}=G(z),
$$

where $G$ is an entire function having an infinite number of simple zeros and no other zeros. For the class of this surfaces we have $P(R) \leqq 4$ from the value distribution theory of two-valued algebroid functions.

We now consider a characterization of ultrahyperelliptic surfaces in terms of the Picard constant. We first have

Theorem A (Ozawa [6]). $P(R)=4$, if and only if there is a non-constant entire function $H(H(0)=0)$, an entire function $F$ and constants $\gamma$ and $\delta$ such that $G$ in (1.1) satisfies

$$
F(z)^{2} G(z)=\left(e^{H(z)}-\gamma\right)\left(e^{H(z)}-\delta\right), \quad \gamma \delta(\gamma-\delta) \neq 0 .
$$

When $P(R)=3$ we have

THEOREM B (Hiromi-Ozawa [1]). If $P(R)=3$, then there are two non-constant entire functions $H$ and $L(H(0)=L(0)=0)$, an entire function $F$ and non-zero constants $\beta_{1}$ and $\beta_{2}$ such that $G$ in (1.1) satisfies

Received June 29, 1992. 


$$
\begin{aligned}
& F(z)^{2} G(z) \\
& =1-2 \beta_{1} e^{H(z)}-2 \beta_{2} e^{L(z)}+{\beta_{1}}^{2} e^{2 H(z)}-2 \beta_{1} \beta_{2} e^{H(z)+L(z)}+{\beta_{2}}^{2} e^{2 L(z)} .
\end{aligned}
$$

Conversely, if $G$ satisfies (1.3), then the ultrahyperelliptic surface $R$ defined by $(1.1)$ is of $P(R) \geqq 3$.

It is quite difficult to determine all ultrahyperelliptic surfaces with $P(R)=3$ and we have not perfectly succeed in it yet. However we have following:

THEOREM C (Ozawa [7]). Let $R$ be the ultrahyperelliptic surface defined by (1.1) with $G$ satısfying (1.3). If $H$ and $L$ are polynomials, then $P(R)=3$ with following four exceptional cases: (i) $H=L$; (ii) $H=2 L, 16 \beta_{1}={\beta_{2}{ }^{2}}^{2}$; (iii) $2 H=L$, $\beta_{1}{ }^{2}=16 \beta_{2}$; (iv) $H=-L, 16 \beta_{1} \beta_{2}=1$. In these exceptional cases we have $P(R)=4$.

From this theorem we conjecture that Theorem $\mathrm{C}$ is also true when $H$ and $L$ in (1.3) are transcendental.

In this paper we shall prove the following:

THEOREM. Let $R$ be the ultrahyperelliptic surfaces defined by (1.1) with $G$ satisfying (1.3). If $H$ and $L$ in (1.3) are transcendental entire functions such that $H(0)=L(0)=0$ and

$$
L(z)=\lambda H(z)+K(z),
$$

where $\lambda$ is a rational number and $K$ is an entire function satısfying

$$
m\left(r, e^{K}\right)=o\left(m\left(r, e^{H}\right)\right), \quad r \rightarrow \infty,
$$

outside a set of finite measure, then $P(R)=3$ with following four exceptional cases: (i) $H=L$; (ii) $H=2 L, 16 \beta_{1}=\beta_{2}{ }^{2}$; (iii) $2 H=L,{\beta_{1}}^{2}=16 \beta_{2}$; (iv) $H=-L$, $16 \beta_{1} \beta_{2}=1$. In these exceptional cases we have $P(R)=4$.

Remark. Hiromi-Ozawa [1] proved this theorem when $\lambda=0$ in (1.4).

We assume that the reader is familiar with the Nevanlinna theory of meromorphic functions and the usual notations such as $T(r, f), N(r, a, f), N_{1}(r, a, f)$, $m(r, f)$ etc. (see e.g. [2]).

\section{§. Lemmas.}

Here we state Lemmas needed in the proofs of our Theorem and Proposition.

Lemma A ([1]). Let $a_{1}, \cdots, a_{n}$ be meromorphic functions and $H$ a nonconstant entire function. Suppose that

$$
T\left(r, a_{\mu}\right)=o\left(m\left(r, e^{H}\right)\right) \quad(r \rightarrow \infty) \quad \mu=1, \cdots, n
$$


hold outside a set of finite measure. Then the equation

$$
\sum_{\mu=1}^{n} a_{\mu}(z) e^{\mu H(z)}=0
$$

cannot hold unless $a_{1} \equiv \cdots \equiv a_{n} \equiv 0$.

LEMMA B ([4]). Let $a_{\mu, \nu}(\mu, \nu=0,1, \cdots, m)$ be meromorphic functions and $H$ and $M$ two non-constant entre functions such that

$$
m\left(r, e^{H}\right) \sim m\left(r, e^{M}\right) \quad \text { and } T\left(r, a_{\mu, \nu}\right)=o\left(m\left(r, e^{H}\right)\right) \quad(r \rightarrow \infty)
$$

hold for $\mu, \nu=0,1, \cdots, m$, outside a set of $r$ of finite measure. Further suppose that

$$
\sum_{\mu, \nu=0}^{m} a_{\mu, \nu}(z) e^{\mu H(z)+\nu M(z)}=0
$$

( I ) If $a_{m, m}(z) \not \equiv 0$, then $a_{m, 0}(z) \equiv a_{0, m}(z) \equiv 0$ and $m\left(r, e^{H+M}\right)=o\left(m\left(r, e^{H}\right)\right)(r \rightarrow$ $\infty)$ outside a set of $r$ of finite measure.

(II) If $a_{\nu, \nu}(z) \equiv 0(\nu=0,1, \cdots, m-1)$ and $a_{m, 0} \neq \equiv$ or $a_{0, m} \neq \equiv$, then $a_{m, m}(z)$ $\equiv 0$ and $m\left(r, e^{H-M}\right)=o\left(m\left(r, e^{H}\right)\right)(r \rightarrow \infty)$ outside $a$ set of $r$ of finite measure.

Let $N_{2}(r, 0, f)$ be the counting function of simple zeros of the indicated function $f$. We can deduce from Nevanlinna's second fundamental theorem that

LEMMA C (cf. [6]). Let $H$ be a non-constant entire function and $a(\not \equiv 0) a$ meromorphic function satisfying

$$
T(r, a)=o\left(m\left(r, e^{H}\right)\right) \quad(r \rightarrow \infty)
$$

outside a set of $r$ of finite measure. Then we have

$$
N_{2}\left(r, 0, e^{I I}-a\right) \sim m\left(r, e^{H}\right) \quad \text { and } N_{1}\left(r, 0, e^{H}-a\right)=o\left(m\left(r, e^{H}\right)\right) \quad(r \rightarrow \infty)
$$

outside a set of finite measure.

From Lemma 4 and Lemma 5 in [3] and Lemma $C$ we can deduce

LEMMA D. Let $H$ and $a_{\jmath}(\jmath=1, \cdots, \mu)$ be entıre functions satisfying

$$
m\left(r, a_{j}\right)=o\left(m\left(r, e^{H}\right)\right) \quad(r \rightarrow \infty) \quad \jmath=1, \cdots, \mu
$$

outside a set of finite measure. If the discriminant of the equation

$$
Q_{\mu}(x):=x^{\mu}+a_{1}(z) x^{\mu-1}+\cdots+a_{\mu}(z)=0
$$

is not identically zero, then we have

(2.1) $N_{2}\left(r, 0, Q_{\mu}\left(e^{H}\right)\right) \sim \mu m\left(r, e^{H}\right) \quad$ and $\quad N_{1}\left(r, 0, Q_{\mu}\left(e^{H}\right)\right)=o\left(m\left(r, e^{H}\right)\right) \quad(r \rightarrow \infty)$ 
outside a set of finite measure.

We can easily deduce

LEMMA E. The discriminant $D$ of the algebraic equation $P(x):=x^{p}+a x+b$ $=0$, where $a$ and $b$ are non-zero constants, is

$$
D=(-1)^{p(p-1) / 2+(p-1)}\left\{(p-1)^{p-1} a^{p}+(-1)^{p-1} p^{p} b^{p-1}\right\} .
$$

Further, if $D$ is zero, then the discriminant of the equation $Q(x)=0$ is not zero, where

$$
P(x)=\{x+p b /(p-1) a\}^{2} Q(x) .
$$

LEMMA F. The discriminant $D$ of the algebraic equation $P(x):=x^{q}+a x^{q-1}+$ $b=0$, where $a$ and $b$ are non-zero constants, is

$$
D=(-1)^{q(q-1) / 2+(q-1)} b^{q-2}\left\{(q-1)^{q-1} a^{q}+(-1)^{q-1} q^{q} b\right\} .
$$

Further, if $D$ is zero, then the discriminant of the equation $Q(x)=0$ is not zero, where

$$
P(x)=\{x+(q-1) a / q\}^{2} Q(x) .
$$

LEMMA G. Let $p$ and $q$ be coprime integers satisfying $p>q>1$. The algebraic equation

$$
x^{p}+a x^{q}+b=0 \quad(a b \neq 0)
$$

has a multiple root $\alpha$, if and only if

$$
D_{0}:=(p-q)^{p-q} q^{q} a^{p}+(-1)^{p-1} p^{p} b^{p-q}=0 .
$$

Then $\alpha$ is only one double root and satisfies

$$
\alpha^{p-q}=-a q / p \text { and } \alpha^{q}=-b p / a(p-q) .
$$

We here note that $D_{0}$ is equal to the discriminant of the algebraic equation (2.2) modulo a non-zero.

\section{§3. Proposition.}

Proposition. Let $H$ and $M$ be non-constant entire functions with $H(0)=$ $M(0)=0$ and $a_{\mu}(\mu=0,1, \cdots, 2 p)$ entire functions satisfying $a_{0} \not \equiv 0, a_{2 p} \not \equiv 0$ and

$$
m\left(r, a_{\mu}\right)=o\left(m\left(r, e^{H}\right)\right) \quad(r \rightarrow \infty) \quad \jmath=0,1, \cdots, 2 p,
$$

outside a set of finite measure, where $p$ is a positive integer. Further we assume that

$$
g(z):=\sum_{\mu=0}^{2 p} a_{\mu}(z) e^{\mu H(z)}
$$


satisfies

$$
N_{2}(r, 0, g) \sim 2 p m\left(r, e^{I I}\right) \quad \text { and } \quad N_{1}(r, 0, g)=o\left(m\left(r, e^{H}\right)\right) \quad(r \rightarrow \infty)
$$

outside a set of finite measure. If the identity

$$
f(z)^{2}\left(e^{p M(z)}-\gamma\right)\left(e^{p M(z)}-\delta\right)=g(z),
$$

holds with a suitable meromorphic function $f$ and two suitable constants $\gamma$ and $\delta$ satisfying $\gamma \delta(\gamma-\delta) \neq 0$, then we have

or

$$
\begin{aligned}
& a_{p}=-a_{2 p}(\gamma+\delta) e^{p(H+M)} / \gamma \delta, \quad a_{0}=a_{2 p} e^{2 p(H+M)} / \gamma \delta \\
& a_{2 p-1} \equiv \cdots \equiv a_{p+1} \equiv a_{p-1} \equiv \cdots \equiv a_{1} \equiv 0, \quad f^{2}=a_{2 p} e^{2 p H} / \gamma \delta
\end{aligned}
$$

$$
\begin{aligned}
& a_{p}=-a_{2 p}(\gamma+\delta) e^{p(H-M)}, \quad a_{0}=a_{2 p} \gamma \delta e^{2 p(H-M)}, \\
& a_{2 p-1} \equiv \cdots \equiv a_{p+1} \equiv a_{p-1} \equiv \cdots \equiv a_{1} \equiv 0, \quad f^{2}=a_{2 p} e^{2 p(H-M)} .
\end{aligned}
$$

Proof. It follows from Lemma $\mathrm{C}$ that

$$
\begin{aligned}
& N_{2}\left(r, 0,\left(e^{p M}-\gamma\right)\left(e^{p M}-\delta\right)\right) \sim 2 p m\left(r, e^{M}\right), \\
& N_{1}\left(r, 0,\left(e^{p M}-\gamma\right)\left(e^{p M}-\delta\right)\right)=o\left(m\left(r, e^{M}\right)\right), \quad(r \rightarrow \infty)
\end{aligned}
$$

outside a set of finite measure. Hence, considering simple zeros and multiple zeros of the both sides of (3.4), from (3.1), (3.2), (3.3), (3.4) and the reasoning of $[4$, p. 298] we can deduce that

$$
\begin{gathered}
m\left(r, e^{M}\right) \sim m\left(r, e^{H}\right), \\
T(r, f)=O\left(m\left(r, e^{H}\right)\right), \quad T\left(r, f^{\prime} / f\right)=o\left(m\left(r, e^{H}\right)\right) \quad(r \rightarrow \infty)
\end{gathered}
$$

outside a set of finite measure. Differentiating both sides of (3.4) and using (3.2) and (3.4) we obtain

$$
\sum_{\mu, \nu=0}^{2 p} b_{\mu, \nu}(z) e^{\mu H(z)+\nu M(z)}=0,
$$

where $b_{2 p, 2 p}=\left(2 f^{\prime} / f+2 p M^{\prime}\right) a_{2 p}-\left(a_{2 p^{\prime}}+2 p a_{2 p} H^{\prime}\right), \quad b_{2 p, 0}=\gamma \delta\left(2 a_{2 p} f^{\prime} / f-a_{2 p^{\prime}}-\right.$ $\left.2 p a_{2 p} H^{\prime}\right), \quad b_{0,2 p}=\left(2 f^{\prime} / f+2 p M^{\prime}\right) a_{0}-a_{0}^{\prime}$ and the others $b_{\mu, \nu}$ are meromorphic functions. It is clear from (3.7) and our assumption (3.1) that

$$
T\left(r, b_{\mu, \nu}\right)=o\left(m\left(r, e^{H}\right)\right) \quad(r \rightarrow \infty) \quad \text { for } \quad \mu, \nu=0,1, \cdots, 2 p
$$

outside a set of finite measure. We apply Lemma B to the identity (3.8).

Suppose that $b_{2 p, 2 p} \neq \equiv 0$. Then (I) of Lemma B yields $b_{2 p, 0} \equiv b_{0,2 p} \equiv 0$ and

$$
m\left(r, e^{H+M}\right)=o\left(m\left(r, e^{H}\right)\right) \quad(r \rightarrow \infty)
$$

outside a set of finite measure. Hence we have $f(z)^{2}=c a_{2 p}(z) e^{2 p H(z)}=$ 
$d a_{0}(z) e^{-2 p M(z)}$ with non-zero constants $c$ and $d$. Substituting these relations into (3.4) and using (3.2) we have

$$
\begin{aligned}
& (1-c \gamma \delta) a_{2 p} e^{2 p H}+a_{2 p-1} e^{(2 p-1) H}+\cdots+a_{p+1} e^{(p+1) H} \\
& +\left(a_{p}+c(\gamma+\delta) a_{2 p} e^{p(H+M)}\right) e^{p H}+a_{p-1} e^{(p-1) H}+\cdots+a_{1} e^{H}+a_{0}-c a_{2 p} e^{2 p(H+M)}=0 .
\end{aligned}
$$

Hence it follows from Lemma A and (3.9) that

$$
\begin{aligned}
& (1-c \gamma \delta) a_{2 p} \equiv 0, \quad a_{2 p-1} \equiv \cdots \equiv a_{p+1} \equiv 0, \quad a_{p}+c(\gamma+\delta) a_{2 p} e^{p(H+M)} \equiv 0, \\
& a_{p-1} \equiv \cdots \equiv a_{1} \equiv 0, \quad a_{0}-c a_{2 p} e^{2 p(H+M)} \equiv 0 .
\end{aligned}
$$

Thus we have (3.5) because of $a_{2 p} \neq \equiv$.

Next suppose that $b_{2 p, 2 p} \equiv 0$. Then we have $f(z)^{2}=c a_{2 p}(z) e^{2 p(H(z)-M(z))}$ with a non-zero constant $c$ and so (3.4) reduces to

$$
\sum_{\mu, \nu=0}^{2 p} c_{\mu, \nu}(z) e^{\mu H(z)+\nu M(z)}=0,
$$

where $c_{2 p, 2 p}=(1-c) a_{2 p}, c_{2 p-1,2 p-1}=\cdots=c_{1,1}=c_{0,0}=0, c_{2 p, 0}=-c \gamma \delta a_{2 p}, c_{0,2 p}=b_{0}$ and the others $c_{\mu, \nu}$ are entire functions satisfying

$$
T\left(r, c_{\mu, \nu}\right)=o\left(m\left(r, e^{H}\right)\right) \quad(r \rightarrow \infty)
$$

outside a set of finite measure. Since $c_{2 p, 0} \neq 0$ and $c_{0,2 p} \not \equiv 0$, (II) of Lemma B implies $(1-c) a_{2 p} \equiv 0$ and

$$
m\left(r, e^{H-M}\right)=o\left(m\left(r, e^{H}\right)\right) \quad(r \rightarrow \infty)
$$

outside a set of finite measure. Hence, since $a_{2 p} \not \equiv 0$ so $c=1$, the identity (3.10) further reduces to

$$
\begin{aligned}
& a_{2 p-1} e^{(2 p-1)(H-M)} e^{(2 p-1) M}+\cdots+a_{p+1} e^{(p+1)(H-M)} e^{(p+1) M} \\
& \quad+\left(a_{p} e^{p(H-M)}+a_{2 p}(\gamma+\delta) e^{2 p(H-M)}\right) e^{p M} \\
& \quad+a_{p-1} e^{(p-1)(H-M)} e^{(p-1) M}+\cdots+a_{1} e^{H-M} e^{M}+a_{0}-a_{2 p} \gamma \delta e^{2 p(H-M)}=0 .
\end{aligned}
$$

Therefore it follows from (3.11) and Lemma A that

$$
\begin{aligned}
& a_{2 p-1} e^{(2 p-1)(H-M)} \equiv \cdots \equiv a_{p+1} e^{(p+1)(H-M)} \equiv 0, \\
& a_{p} e^{p(H-M)}+a_{2 p}(\gamma+\delta) e^{2 p(H-M)} \equiv 0, \\
& a_{p-1} e^{(p-1)(H-M)} \equiv \cdots \equiv a_{1} e^{H-M} \equiv 0, \quad a_{0}-a_{2 p} \gamma \delta e^{2 p(H-M)} \equiv 0 .
\end{aligned}
$$

Hence we have (3.6).

Thus the proof of our Proposition is complete. 


\section{§4. Proof of Theorem.} (1.4).

It is sufficient to consider only the cases only the cases $\lambda=0$ and $|\lambda| \geqq 1$ in

Eight main cases are to be considered.

(A) If $\lambda=0$ in (1.4), then our assumption (1.5) and Theorem $C$ in HiromiOzawa [1] imply $P(R)=3$.

(B) Assume that $\lambda=1$ in (1.4). Then (1.3) reduces to

$$
g(z):=F(z)^{2} G(z)=\left(\beta_{1}-\beta_{2} e^{K(z)}\right)^{2} e^{2 H(z)}+2\left(\beta_{1}+\beta_{2} e^{K(z)}\right) e^{H(z)}+1 .
$$

If $K \equiv$ const., then we have $K \equiv 0$ and so $H \equiv L$, because of $H(0)=L(0)=0$ in (1.4), and (4.1) reduces to

and

$$
g=F^{2} G=4 \beta_{1}\left(e^{H / 2}-\left(2 \beta_{1}{ }^{1 / 2}\right)^{-1}\right)\left(e^{H / 2}+\left(2 \beta_{1}{ }^{1 / 2}\right)^{-1}\right), \quad \text { if } \quad \beta_{1}=\beta_{2},
$$

$$
g=F^{2} G=\left(\beta_{1}-\beta_{2}\right)^{2}\left(e^{H}-\gamma_{1}\right)\left(e^{H I}-\gamma_{2}\right), \quad \text { if } \quad \beta_{1} \neq \beta_{2},
$$

where $\gamma_{1}=-\left(\beta_{1}{ }^{1 / 2}+\beta_{2}{ }^{1 / 2}\right)^{2} /\left(\beta_{1}-\beta_{2}\right)^{2}$ and $\gamma_{2}=-\left(\beta_{1}{ }^{1 / 2}-\beta_{2}{ }^{1 / 2}\right)^{2} /\left(\beta_{1}-\beta_{2}\right)^{2}$. Hence it follows from Theorem $\mathrm{A}$ that $P(R)=4$. So this case corresponds the exceptional case $(\mathrm{i})$.

If $K \neq \equiv$ const., then $\beta_{1}-\beta_{2} e^{K} \neq \equiv$. The discriminant of the equation $Q(x)$ : $=\left(\beta_{1}-\beta_{2} e^{K(z)}\right)^{2} x^{2}+2\left(\beta_{1}+\beta_{2} e^{K(z)}\right) x+1=0$ is $16 \beta_{1} \beta_{2} e^{K(z)} \neq 0$ and $g(z)=Q\left(e^{H(z)}\right)$ by (4.1). Hence Lemma D yields

$$
N_{2}(r, 0, g) \sim 2 m\left(r, e^{H}\right) \quad \text { and } \quad N_{1}(r, 0, g)=o\left(m\left(r, e^{H}\right)\right) \quad(r \rightarrow \infty)
$$

outside a set of finite measure. Now assume $P(R)=4$. Then it follows from Theorem A that there is a non-constant entire function $M$, a meromorphic function $f$ and constants $\gamma$ and $\delta$ such that

$$
f(z)^{2}\left(e^{M(z)}-\gamma\right)\left(e^{M(z)}-\delta\right)=g(z), \quad M(0)=0, \quad \gamma \delta(\gamma-\delta) \neq 0 .
$$

Here, since (1.5) and (4.2) hold, we can apply our Proposition to the identity (4.3) with (4.1). Put (4.1) into $g(z)=a_{2}(z) e^{2 H(z)}+a_{1}(z) e^{H(z)}+a_{0}(z)$. Then Proposition implies that

$$
a_{1}(z)=-a_{2}(z)(\gamma+\delta) e^{H(z)+M(z)} / \gamma \delta, \quad a_{0}(z)=a_{2}(z) e^{2(H(z)+M(z))} / \gamma \delta
$$

or

$$
a_{1}(z)=-a_{2}(z)(\gamma+\delta) e^{H(z)-M(z)}, \quad a_{0}(z)=a_{2}(z) \gamma \delta e^{2(H(z)-M(z))},
$$

that is,

$$
\begin{aligned}
2\left(\beta_{1}+\beta_{2} e^{K(z)}\right) & =-\left(\beta_{1}-\beta_{2} e^{K(z)}\right)^{2}(\gamma+\delta) e^{H(z)+M(z)} / \gamma \delta, \\
1 & =\left(\beta_{1}-\beta_{2} e^{K(z)}\right)^{2} e^{2(H(z)+M(z))} / \gamma \delta
\end{aligned}
$$

or 


$$
\begin{aligned}
2\left(\beta_{1}+\beta_{2} e^{K(z)}\right) & =-\left(\beta_{1}-\beta_{2} e^{K(z)}\right)^{2}(\gamma+\delta) e^{H(z)-M(z)}, \\
1 & =\left(\beta_{1}-\beta_{2} e^{K(z)}\right)^{2} \gamma \delta e^{2(H(z)-M(z))} .
\end{aligned}
$$

This is a contradiction, because the function $\beta_{1}-\beta_{2} e^{K(z)}$ has simple zeros since $K \not \equiv$ const..

Thus we have showed that if $K \not \equiv$ const., then $P(R) \neq 4$ and hence, by Theorem B, $P(R)=3$.

(C) Assume that $\lambda=2$ in (1.4). Then (1.3) is

$$
g:=F^{2} G=\beta_{2}{ }^{2} e^{2 K} e^{4 H}-2 \beta_{1} \beta_{2} e^{K} e^{3 H}+\left(\beta_{1}{ }^{2}-2 \beta_{2} e^{K}\right) e^{2 H}-2 \beta_{1} e^{H}+1 .
$$

If $K \equiv$ const. and ${\beta_{1}}^{2}=16 \beta_{2}$, then $K \equiv 0$, so $L \equiv 2 H$ and (4.4) reduces to

$$
g=F^{2} G=\left(\beta_{1}{ }^{4} / 16^{2}\right)\left(e^{H}-4 \beta_{1}{ }^{-1}\right)^{2}\left(e^{H}-4(1+\sqrt{2})^{2} \beta_{1}{ }^{-1}\right)\left(e^{H}-4(1-\sqrt{2})^{2} \beta_{1}{ }^{-1}\right) .
$$

Hence we have

$$
\left\{F\left(16 / \beta_{1}{ }^{-2}\right)\left(e^{H}-4 \beta_{1}{ }^{-1}\right)^{-1}\right\}^{2} G=\left(e^{I I}-4(1+\sqrt{2})^{2} \beta_{1}{ }^{-1}\right)\left(e^{H}-4(1-\sqrt{2})^{2} \beta_{1}^{-1}\right)
$$

and so theorem A implies $P(R)=4$. This case corresponds the exceptional case (iii).

If $K \not \equiv$ const. or $\beta_{1}{ }^{2} \neq 16 \beta_{2}$, then (4.4) is rewritten as follows :

$$
g(z)=\beta_{2}{ }^{2} e^{2 K(2)} g_{1}\left(e^{H(z) / 2}\right) g_{2}\left(e^{H(z) / 2}\right) g_{3}\left(e^{H(z) / 2}\right) g_{4}\left(e^{H(z) / 2}\right),
$$

where

$$
\begin{aligned}
& g_{1}(x)=x^{2}+\left(\beta_{1} / \beta_{2}\right)^{1 / 2} e^{-K / 2} x-\beta_{2}^{-1 / 2} e^{-K / 2}, \\
& g_{2}(x)=x^{2}-\left(\beta_{1} / \beta_{2}\right)^{1 / 2} e^{-K / 2} x+\beta_{2}^{-1 / 2} e^{-K / 2}, \\
& g_{3}(x)=x^{2}-\left(\beta_{1} / \beta_{2}\right)^{1 / 2} e^{-K / 2} x-\beta_{2}^{-1 / 2} e^{-K / 2}, \\
& g_{4}(x)=x^{2}+\left(\beta_{1} / \beta_{2}\right)^{1 / 2} e^{-K / 2} x+\beta_{2}^{-1 / 2} e^{-K / 2} .
\end{aligned}
$$

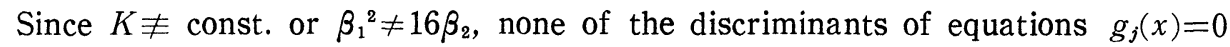
$(j=1, \cdots, 4)$ vanish and these equations have no common algebroid solution. Hence the discriminant of the equation $g^{*}(x)=0$ is not identically zero, where $g^{*}(x)=g_{1}(x) \cdots g_{4}(x)$, and so (4.5) and Lemma D imply

$$
N_{2}(r, 0, g) \sim 4 m\left(r, e^{H}\right) \quad \text { and } N_{1}(r, 0, g)=o\left(m\left(r, e^{H}\right)\right) \quad(r \rightarrow \infty)
$$

outside a set of finite measure. Now assume $P(R)=4$. Then it follows from Theorem A that there is a non-constant entire function $M$, a meromorphic function $f$ and two constants $\gamma$ and $\delta$ such that

$$
f(\boldsymbol{z})^{2}\left(e^{2 M(z)}-\gamma\right)\left(e^{2 M(z)}-\delta\right)=g(z), \quad M(0)=0, \quad \gamma \delta(\gamma-\delta) \neq 0 .
$$

Then it follows from (1.5), (4.5), (4.6) and our Proposition that the coefficients of $e^{3 H}$ and $e^{H}$ in (4.4) must be identically zero, that is, 


$$
2 \beta_{1} \beta_{2} e^{K(z)} \equiv-2 \beta_{1} \equiv 0,
$$

which contradicts $\beta_{1} \neq 0$.

Thus we have showed that if $K \not \equiv$ const. or $\beta_{1}{ }^{2} \neq 16 \beta_{2}$, then $P(R) \neq 4$.

(D) Assume that $\lambda=-1$ in (1.5). Then (1.3) is

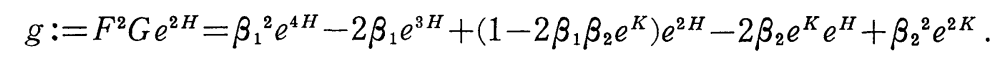

If $K \equiv$ const. and $16 \beta_{1} \beta_{2}=1$, then $K \equiv 0$ and so $L \equiv-H$, and if follows from (4.7) that

$$
\left\{F e^{H} \beta_{1}^{-1}\left(e^{H}-1 / 4 \beta_{1}\right)^{-1}\right\}^{2} G=\left(e^{H}-(1+\sqrt{2})^{2} / 4 \beta_{1}\right)\left(e^{H}-(1-\sqrt{2})^{2} / 4 \beta_{1}\right) .
$$

Hence Theorem A implies $P(R)=4$. 'This case corresponds the exceptional case (iv).

If $K \not \equiv$ const. or $16 \beta_{1} \beta_{2} \neq 1$, then (4.7) is rewritten as follows :

$$
g(z)=\beta_{1}^{2} g_{1}\left(e^{H(z) / 2}\right) g_{2}\left(e^{H(z) / 2}\right) g_{3}\left(e^{H(z) / 2}\right) g_{4}\left(e^{H(z) / 2}\right),
$$

where

$$
\begin{aligned}
& g_{1}(x)=x^{2}+\beta_{1}^{-1 / 2} x-\left(\beta_{2} / \beta_{1}\right)^{1 / 2} e^{K / 2}, \\
& g_{2}(x)=x^{2}-\beta_{1}^{-1 / 2} x+\left(\beta_{2} / \beta_{1}\right)^{1 / 2} e^{K / 2}, \\
& g_{3}(x)=x^{2}-\beta_{1}^{-1 / 2} x-\left(\beta_{2} / \beta_{1}\right)^{1 / 2} e^{K / 2}, \\
& g_{4}(x)=x^{2}+\beta_{1}^{-1 / 2} x+\left(\beta_{2} / \beta_{1}\right)^{1 / 2} e^{K / 2} .
\end{aligned}
$$

Since $K \not \equiv$ const. or $16 \beta_{1} \beta_{2} \neq 1$, none of the discriminants of equations $g_{j}(x)=0$ $(\jmath=1, \cdots, 4)$ vanish and these equations have no common algebroid solution. Hence the discriminant of the equation $g^{*}(x)=0$ is not identically zero, where $g^{*}(x)=g_{1}(x) \cdots g_{4}(x)$, and so (4.8) and Lemma D imply

$$
N_{2}(r, 0, g) \sim 4 m\left(r, e^{H}\right) \quad \text { and } N_{1}(r, 0, g)=o\left(m\left(r, e^{H}\right)\right) \quad(r \rightarrow \infty)
$$

outside a set of finite measure. Now assume $P(R)=4$. Then it follows from Theorem A that there is a non-constant entire function $M$, a meromorphic function $f$ and two constants $\gamma$ and $\delta$ such that

$$
f(z)^{2}\left(e^{2 M(z)}-\gamma\right)\left(e^{2 M(z)}-\delta\right)=g(z), \quad M(0)=0, \quad \gamma \delta(\gamma-\delta) \neq 0 .
$$

Then it follows from (1.5), (4.8), (4.9) and our Proposition that the coefficient $-2 \beta_{1}$ of $e^{3 H}$ in (4.7) must be identically zero, which contradicts $\beta_{1} \neq 0$.

Thus we have showed that if $K \not \equiv$ const. or $16 \beta_{1} \beta_{2} \neq 1$, then $P(R) \neq 4$.

(E) Assume that $\lambda=p$ is an integer and $p>2$ in (1.4). Then (1.3) is

where

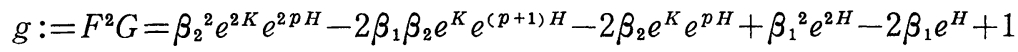

$$
\begin{aligned}
& =\beta_{2}{ }^{2} e^{2 K} g_{1}\left(e^{H / 2}\right) g_{2}\left(e^{H / 2}\right) g_{3}\left(e^{H / 2}\right) g_{4}\left(e^{H / 2}\right),
\end{aligned}
$$




$$
\begin{aligned}
& g_{1}(x)=x^{p}+\left(\beta_{1} / \beta_{2}\right)^{1 / 2} e^{-K / 2} x-\beta_{2}^{-1 / 2} e^{-K / 2}, \\
& g_{2}(x)=x^{p}-\left(\beta_{1} / \beta_{2}\right)^{1 / 2} e^{-K / 2} x+\beta_{2}^{-1 / 2} e^{-K / 2}, \\
& g_{3}(x)=x^{p}-\left(\beta_{1} / \beta_{2}\right)^{1 / 2} e^{-K / 2} x-\beta_{2}^{-1 / 2} e^{-K / 2}, \\
& g_{4}(x)=x^{p}+\left(\beta_{1} / \beta_{2}\right)^{1 / 2} e^{-K / 2} x+\beta_{2}^{-1 / 2} e^{-K / 2} .
\end{aligned}
$$

Suppose that $K \neq \equiv$ const. or $(p-1)^{2 p-2} \beta_{1}{ }^{p} \neq p^{2 p} \beta_{2}$. Then it follows from Lemma $\mathrm{E}$ that none of the discriminants of equations $g_{j}(x)=0(j=1, \cdots, 4)$ vanish. Hence, since these equations have no common algebroid solution, (4.10) and Lemma D imply

$$
N_{2}(r, 0, g) \sim 2 p m\left(r, e^{H}\right) \quad \text { and } \quad N_{1}(r, 0, g)=o\left(m\left(r, e^{H}\right)\right) \quad(r \rightarrow \infty)
$$

outside a set of finite measure. Since the coefficient of $e^{(p+1) H(z)}$ in (4.10) is $-2 \beta_{1} \beta_{2} e^{K(z)}$, which is not identically zero, our Proposition and Theorem A imply that $P(R) \neq 4$ if $K \neq$ const. or $(p-1)^{2 p-2} \beta_{1}{ }^{p} \neq p^{2 p} \beta_{2}$.

Next we suppose that $K \equiv$ const. and $(p-1)^{2 p-2} \beta_{1}{ }^{p}=p^{2 p} \beta_{2}$. Then it follows from Lemma $\mathrm{E}$ that two of discriminants $D$, of equations $g_{j}(x)=0(j=1, \cdots, 4)$ are zero and the others are not zero. For example, $D_{1}=D_{3}=0$ and $D_{2}= \pm D_{4} \neq 0$ if $(p-1)^{p-1}{\beta_{1}}^{p / 2}=p^{p}{\beta_{2}}^{1 / 2}$ and $p$ is even. Then (4.10) reduces to

$$
g=F^{2} G=\beta_{2}{ }^{2}\left(e^{H}-\alpha\right)^{2} g_{0}\left(e^{H}\right)
$$

and so we have

$$
\left\{F(z) \beta_{2}^{-1}\left(e^{H(z)}-\alpha\right)^{-1}\right\}^{2} G(z)=g_{0}\left(e^{H(z)}\right),
$$

where $\alpha=p^{2} / \beta_{1}(p-1)^{2}$ and

$$
\begin{aligned}
g_{0}(y):=y^{2 p-2} & +2 \alpha y^{2 p-3}+\cdots+(p-1) \alpha^{p-2} y^{p} \\
& +a_{p-1} y^{P-1}+\cdots+a_{1} y+(p-1)^{4}{\beta_{1}}^{2} / p^{4}{\beta_{2}}^{2},
\end{aligned}
$$

where $a_{1}, \cdots, a_{p-1}$ are suitable constants. It follows from Lemma $\mathrm{E}$ that the discriminant of equation $g_{0}(y)=0$ is not zero. So Lemma D implies

$$
\begin{aligned}
& N_{2}\left(r, 0, g_{0}\left(e^{H}\right)\right) \sim(2 p-2) m\left(r, e^{H}\right), \\
& N_{1}\left(r, 0, g_{0}\left(e^{H}\right)\right)=o\left(m\left(r, e^{H}\right)\right) \quad(r \rightarrow \infty)
\end{aligned}
$$

outside a set of finite measure. Since $2 p-3>p-1$ and the coefficient of $e^{(2 p-3) H(z)}$ in $g_{0}\left(e^{H(z)}\right)$ is $2 \alpha$, which is not zero, it follows from (4.12), Theorem A and our Proposition that $P(R) \neq 4$ if $K \equiv$ const. and $(p-1)^{2 p-2} \beta_{1}{ }^{p}=p^{2 p} \beta_{2}$.

Thus we have proved that $P(R) \neq 4$ if $\lambda$ is an integer greater than 2 .

(F) Assume that $\lambda=-q+1$ is an integer and $\lambda<-1$, that is, $q>2$ in (1.4). Then from (1.3) we have 


$$
\begin{aligned}
& g:=\left(F e^{(q-1) H}\right)^{2} G \\
& =\beta_{1}{ }^{2} e^{2 q H}-2 \beta_{1} e^{(2 q-1) H}+e^{(2 q-2) H}-2 \beta_{1} \beta_{2} e^{K} e^{q H}-2 \beta_{2} e^{K} e^{(q-1) H}+\beta_{2}{ }^{2} e^{2 K} \\
& =\beta_{1}{ }^{2} g_{1}\left(e^{H / 2}\right) g_{2}\left(e^{H / 2}\right) g_{3}\left(e^{H / 2}\right) g_{4}\left(e^{H / 2}\right),
\end{aligned}
$$

where

$$
\begin{aligned}
& g_{1}(x)=x^{q}+\beta_{1}^{-1 / 2} x^{q-1}-\left(\beta_{2} / \beta_{1}\right)^{1 / 2} e^{K / 2}, \\
& g_{2}(x)=x^{q}-\beta_{1}^{-1 / 2} x^{q-1}+\left(\beta_{2} / \beta_{1}\right)^{1 / 2} e^{K / 2}, \\
& g_{3}(x)=x^{q}-\beta_{1}^{-1 / 2} x^{q-1}-\left(\beta_{2} / \beta_{1}\right)^{1 / 2} e^{K / 2}, \\
& g_{4}(x)=x^{q}+\beta_{1}^{-1 / 2} x^{q-1}+\left(\beta_{2} / \beta_{1}\right)^{1 / 2} e^{K / 2} .
\end{aligned}
$$

Suppose that $K \neq$ const. or $q^{2 q} \beta_{1}^{q-1} \beta_{2} \neq(q-1)^{2 q-2}$. Then it follows from Lemma $\mathrm{F}$ that none of the discriminants of equations $g_{j}(x)=0(j=1, \cdots, 4)$ vanish. Hence, since these equations have no common algebroid solution, from Lemma $\mathrm{D}$ we have

$$
N_{2}(r, 0, g) \sim 2 q m\left(r, e^{H}\right) \quad \text { and } N_{1}(r, 0, g)=o\left(m\left(r, e^{H}\right)\right) \quad(r \rightarrow \infty)
$$

outside a set of finite measure. Further the coefficient of $e^{(2 q-1) H(z)}$ in (4.13) is $-2 \beta_{1}$, which is not zero. Hence we have $P(R) \neq 4$ from our Proposotion and Theorem A.

Next we suppose that $K \equiv$ const. and $q^{2 q}{\beta_{1}}^{q-1} \beta_{2}=(q-1)^{2 q-2}$. Then it follows from Lemma $\mathrm{F}$ that two of discriminants $D_{j}$ of equations $g_{j}(x)=0(j=1, \cdots, 4)$ are zero and the others are not zero. For example, $D_{2}=D_{4}=0$ and $D_{3}= \pm D_{1}$ $\neq 0$ if $q^{q} \beta_{1}{ }^{(q-1) / 2}{\beta_{2}}^{1 / 2}=(q-1)^{q-1}$ and $q$ is even. Then (4.13) reduces to

$$
g=\left(F e^{(q-1) H}\right)^{2} G=\beta_{1}{ }^{2}\left(e^{H}-\beta\right)^{2} g_{0}\left(e^{H}\right)
$$

and so we have

$$
\left\{F(z) e^{(q-1) H(z)} \beta_{1}^{-1}\left(e^{H(z)}-\beta\right)^{-1}\right\}^{2} G(z)=g_{0}\left(e^{H(z)}\right)
$$

where $\beta=(q-1)^{2} / q^{2} \beta_{1}$ and

$$
g_{0}(y):=y^{2 q-2}-\left(2(2 q-1) / \beta_{1} q^{2}\right) y^{2 q-3}+a_{2 q-4} y^{2 q-4}+\cdots+a_{1} y+\beta_{2} \beta^{q-3} / q^{2}{\beta_{1}}^{2},
$$

where $a_{1}, \cdots, a_{2 q-4}$ are suitable constants. It follows from Lemma $F$ that the discriminant of equation $g_{0}(y)=0$ is not zero. So Lemma D implies

$$
\begin{aligned}
& N_{2}\left(r, 0, g_{0}\left(e^{H}\right)\right) \sim(2 q-2) m\left(r, e^{H}\right), \\
& N_{1}\left(r, 0, g_{0}\left(e^{H}\right)\right)=o\left(m\left(r, e^{H}\right)\right) \quad(r \rightarrow \infty)
\end{aligned}
$$

outside a set of finite measure. Further $2 q-3>q-1$ and the coefficient of $e^{(2 q-3) H(z)}$ in $g_{0}\left(e^{H(z)}\right)$ is $-2(2 q-1) / \beta_{1} q^{2}$, which is not zero. Hence we have $P(R) \neq 4$. 
Thus we have showed that $P(R) \neq 4$ if $\lambda$ is an integer less than -1 .

$(G)$ Assume that $\lambda$ is a rational number greater than one in (1.4) and is not an integer. We put $\lambda=p / q$, where $p$ and $q(q>1)$ are coprime integer Then (1.3) is

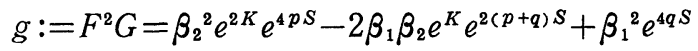

$$
\begin{aligned}
& -2 \beta_{2} e^{K} e^{2 p S}-2 \beta_{1} e^{2 q S}+1 \\
& =\beta_{2}{ }^{2} e^{2 K} g_{1}\left(e^{S}\right) g_{2}\left(e^{S}\right) g_{3}\left(e^{S}\right) g_{4}\left(e^{S}\right),
\end{aligned}
$$

where $S(z)=H(z) / 2 q$ and

$$
\begin{aligned}
& g_{1}(x)=x^{p}+\left(\beta_{1} / \beta_{2}\right)^{1 / 2} e^{-K / 2} x^{q}-\beta_{2}^{-1 / 2} e^{-K / 2}, \\
& g_{2}(x)=x^{p}-\left(\beta_{1} / \beta_{2}\right)^{1 / 2} e^{-K / 2} x^{q}+\beta_{2}^{-1 / 2} e^{-K / 2}, \\
& g_{3}(x)=x^{p}-\left(\beta_{1} / \beta_{2}\right)^{1 / 2} e^{-K / 2} x^{q}-\beta_{2}^{-1 / 2} e^{-K / 2}, \\
& g_{4}(x)=x^{p}+\left(\beta_{1} / \beta_{2}\right)^{1 / 2} e^{-K / 2} x^{q}+\beta_{2}^{-1 / 2} e^{-K / 2} .
\end{aligned}
$$

Suppose that $K \neq$ const. or $(p-q)^{2(p-q)} q^{2 q} \beta_{1}{ }^{p} \neq p^{2 p} \beta_{2}^{q}$. Then it follows from Lemma $\mathrm{G}$ that none of the discriminants of equations $g_{j}(x)=0(j=1, \cdots, 4)$ vanish. Hence, since these equations have no common algebroid solution, (4.15) and Lemma D imply

$$
N_{2}(r, 0, g) \sim 4 p m\left(r, e^{S}\right) \quad \text { and } \quad N_{1}(r, 0, g)=o\left(m\left(r, e^{S}\right)\right) \quad(r \rightarrow \infty)
$$

outside a set of finite measure. Hence it follows from our Proposition and Theorem A that $P(R) \neq 4$, because the coefficient of $e^{2(p+q) S(z)}$ in $(4.15)$ is $-2 \beta_{1} \beta_{2} e^{K(z)}$, which is not identically zero.

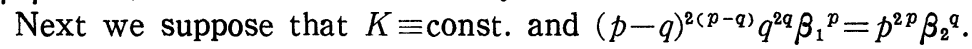

Further, if $q=p-1$, that is, $p=q+1>2$, then the argument in the case $(\mathrm{F})$ is applicable to this case. So we can deduce that $P(R) \neq 4$ in this case.

If $p>q+1$, then $4 p-2>2 p+2 q$ and it follows from Lemma $G$ that two of discriminants $D$, of equations $g_{j}(x)=0(j=1, \cdots, 4)$ are identically zero and the others are not identically zero. (4.15) reduces to

$$
g=F^{2} G={\beta_{1}}^{2}\left\{e^{2 S}-(\alpha+\beta) e^{S}+\alpha \beta\right\}^{2} g_{0}\left(e^{S}\right)
$$

and so we have

$$
\left\{F(z) \beta_{1}^{-1}\left(e^{2 S(z)}-(\alpha+\beta) e^{S(z)}+\alpha \beta\right)^{-1}\right\}^{2} G(z)=g_{0}\left(e^{S(z)}\right),
$$

where $\alpha$ and $\beta$ satisfy one of the following six cases:

(a) if $p$ is even, $q$ is odd and $D_{1}=D_{3}=0$, then

$$
\begin{aligned}
& \alpha^{p-q}=-p q^{-1}\left(\beta_{1} / \beta_{2}\right)^{1 / 2}, \quad \alpha^{q}=p(p-q)^{-1} \beta_{1}{ }^{1 / 2}, \\
& \beta^{p-q}=p q^{-1}\left(\beta_{1} / \beta_{2}\right)^{1 / 2}, \quad \beta^{q}=-p(p-q)^{-1}{\beta_{1}}^{1 / 2},
\end{aligned}
$$


ULTRAHYPERELLIPTIC SURFACE WITH PICARD CONSTANT THREE

(b) if $p$ is even, $q$ is odd and $D_{2}=D_{4}=0$, then

$$
\begin{aligned}
& \alpha^{p-q}=p q^{-1}\left(\beta_{1} / \beta_{2}\right)^{1 / 2}, \quad \alpha^{q}=p(p-q)^{-1} \beta_{1}{ }^{1 / 2}, \\
& \beta^{p-q}=-p q^{-1}\left(\beta_{1} / \beta_{2}\right)^{1 / 2}, \quad \beta^{q}=-p(p-q)^{-1} \beta_{1}^{1 / 2},
\end{aligned}
$$

(c) if $p$ is odd, $q$ is even and $D_{1}=D_{2}=0$, then

$$
\begin{aligned}
& \alpha^{p-q}=-p q^{-1}\left(\beta_{1} / \beta_{2}\right)^{1 / 2}, \quad \alpha^{q}=p(p-q)^{-1}{\beta_{1}{ }^{1 / 2},} \\
& \beta^{p-q}=p q^{-1}\left(\beta_{1} / \beta_{2}\right)^{1 / 2}, \quad \beta^{q}=p(p-q)^{-1}{\beta_{1}{ }^{1 / 2},}^{1 / 2}
\end{aligned}
$$

(d) if $p$ is odd, $q$ is even and $D_{3}=D_{4}=0$, then

$$
\begin{aligned}
& \alpha^{p-q}=p q^{-1}\left(\beta_{1} / \beta_{2}\right)^{1 / 2}, \quad \alpha^{q}=-p(p-q)^{-1} \beta_{1}{ }^{1 / 2}, \\
& \beta^{p-q}=-p q^{-1}\left(\beta_{1} / \beta_{2}\right)^{1 / 2}, \quad \beta^{q}=-p(p-q)^{-1}{\beta_{1}{ }^{1 / 2},}^{1 / 2}
\end{aligned}
$$

(e) if $p$ is odd, $q$ is odd and $D_{1}=D_{4}=0$, then

$$
\begin{array}{ll}
\alpha^{p-q}=-p q^{-1}\left(\beta_{1} / \beta_{2}\right)^{1 / 2}, & \alpha^{q}=p(p-q)^{-1}{\beta_{1}{ }^{1 / 2},}, \\
\beta^{p-q}=-p q^{-1}\left(\beta_{1} / \beta_{2}\right)^{1 / 2}, & \beta^{q}=-p(p-q)^{-1}{\beta_{1}{ }^{1 / 2},}^{2}
\end{array}
$$

(f) if $p$ is odd, $q$ is odd and $D_{2}=D_{3}=0$, then

$$
\begin{array}{ll}
\alpha^{p-q}=p q^{-1}\left(\beta_{1} / \beta_{2}\right)^{1 / 2}, & \alpha^{q}=p(p-q)^{-1}{\beta_{1}{ }^{1 / 2},}^{p-q} \\
\beta^{p-q}=p q^{-1}\left(\beta_{1} / \beta_{2}\right)^{1 / 2}, & \beta^{q}=-p(p-q)^{-1}{\beta_{1}}^{1 / 2}
\end{array}
$$

and

$$
g_{0}(y):=y^{4 p-4}-2(\alpha+\beta) y^{4 p-5}+(\alpha-\beta)^{2} y^{4 p-6}+a_{4 p-7} y^{4 p-7}+\cdots+a_{1} y+a_{0},
$$

where $a_{4 p-7}, \cdots, a_{0}$ are suitable constants. It follows from Lemma $G$ that the discriminant of equation $g_{0}(y)=0$ is not identically zero. So Lemma D implies

$$
\begin{aligned}
& N_{2}\left(r, 0, g_{0}\left(e^{S}\right)\right) \sim(4 p-4) m\left(r, e^{S}\right), \\
& N_{1}\left(r, 0, g_{0}\left(e^{S}\right)\right)=o\left(m\left(r, e^{S}\right)\right) \quad(r \rightarrow \infty)
\end{aligned}
$$

outside a set of finite measure. Further $4 p-6>2 p-2$ and the coefficients of $e^{(4 p-5) S(z)}$ and $e^{(4 p-6) S(z)}$ in $g_{0}\left(e^{S(z)}\right)$ are $-2(\alpha+\beta)$ and $(\alpha-\beta)^{2}$, respectively, which are not simultaneously zero, because $\alpha \neq 0$ and $\beta \neq 0$. Hence we have also $P(R) \neq 4$.

$(\mathrm{H})$ Assume that $\lambda$ is a rational number less than -1 in (1.4) and is not an integer. We can put $\lambda=-q /(p-q)$, where $p$ and $q$ are coprime integer such that $2 q .>p>q+1>1$. Then from (1.3) we have

$$
\begin{aligned}
g & :=\left(F e^{2 q S}\right)^{2} G={\beta_{1}}^{2} e^{4 p S}-2 \beta_{1} e^{2(p+q) S}+e^{4 q S}-2 \beta_{1} \beta_{2} e^{K} e^{2 p S}-2 \beta_{2} e^{K} e^{2 q S}+\beta_{2}{ }^{2} e^{2 K} \\
& =\beta_{1}{ }^{2} g_{1}\left(e^{S}\right) g_{2}\left(e^{S}\right) g_{3}\left(e^{S}\right) g_{4}\left(e^{S}\right),
\end{aligned}
$$


where $S(z)=H(z) / 2(p-q)$ and

$$
\begin{aligned}
& g_{1}(x)=x^{p}+\beta_{1}^{-1 / 2} x^{q}-\left(\beta_{2} / \beta_{1}\right)^{1 / 2} e^{K / 2}, \\
& g_{2}(x)=x^{p}-\beta_{1}^{-1 / 2} x^{q}+\left(\beta_{2} / \beta_{1}\right)^{1 / 2} e^{K / 2}, \\
& g_{3}(x)=x^{p}-\beta_{1}^{-1 / 2} x^{q}-\left(\beta_{2} / \beta_{1}\right)^{1 / 2} e^{K / 2}, \\
& g_{4}(x)=x^{p}+\beta_{1}{ }^{-1 / 2} x^{q}+\left(\beta_{2} / \beta_{1}\right)^{1 / 2} e^{K / 2} .
\end{aligned}
$$

Therefore the same argument in the case $(G)$ leads to $P(R) \neq 4$ in this case.

Thus the proof of our Theorem is complete.

\section{REFERENCES}

[1] Hiromi, G. and Ozawa, M., On the existence of analytic mappings between two ultrahyperelliptic surfaces, Kōdai Math. Sem. Rep. 17 (1965), 281-306.

[2] Nevanlinna, R., Analytic Functions, Springer-Verlag, New York, Heidelberg, Berlin, 1970, pp. 373.

[3] NinNo, K., On reguraly branched three-sheeted covering Riemann surfaces, Kōdaı Math. Sem. Rep. 18 (1966), 229-250.

[4] Nino, K., On the functional equation $\left(e^{M}-\gamma\right)^{k}\left(e^{H}-\delta\right)^{n-k}=f^{m}\left(e^{I I}-\sigma\right)^{k}\left(e^{I I}-\tau\right)^{m-k}$, Aquationes Mathematicae 22 (1981), 293-301.

[5] Ozawa, M., On complex analytic mappings, Kōdai Math. Sem. Rep. 17 (1965), 93-102.

[6] Ozawa, M., On ultrahyperelliptic surfaces, Kōdai Math. Sem. Rep. 17 (1965), 103-108.

[7] Ozawa, M., On an ultrahyperelliptic surface whose Picard's constant is three, Kōdai Math. Sem. Rep. 19 (1967), 245-256.

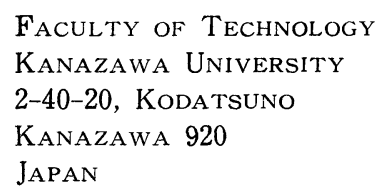

